\title{
МОДЕЛИРОВАНИЕ ТЕЧЕНИЯ ГАЗА В КАМЕРЕ ЖРД С УЧЕТОМ ЕЕ ОХЛАЖДЕНИЯ
}

\author{
И.Д. Дубровский, В.Л. Бучарский
}

Днепровский нащиональный университет имени Олеся Гончара, пр. Гагарина, 72, г. Днепр, 49010, Украина

Розглянуто питання про одновимірний рух газу в камері РРД з урахуванням його теплообміну зі стінкою камери та $з$ охолоджувальною рідиною. Наведено результати розрахунку охолодження камери РРД, отримані шляхом чисельного розв'язку системи диференціальних рівнянь газодинаміки, сумішеної 3 рівнянням теплового балансу. Аналіз отриманих результатів дозволяє стверджувати про можливість застосування запропонованого методу на практиці.

Ключові слова: рідинний ракетний двигун, теплообмін, чисельні методи.

The problem of one-dimensional motion of gas in thecombustion chamber of the liquid rocket engine, taking into account its heat exchange with the chamber wall and with the cooling liquid, is considered in the article. The results of calculating the cooling of the chamber obtained by numerically solving the system of differential equations of gas dynamics combined with the heat balance equation are presented. The analysis of the results allows to assert about the possibility of applying the proposed method in practice.

Keywords: liquid rocket engine, heat transfer, numerical methods.

Рассматрен вопрос об одномерном движении газа в камере ЖРД с учетом его теплообмена со стенкой камеры и с охлаждающей жидкостью. Приведены результаты расчета охлаждения камеры ЖРД, полученные путем численного решения системы дифференциальных уравнений газодинамики, совмещенной с уравнением теплового баланса. Анализ полученных результатов позволяет утверждать о возможности применения предложенного метода на практике.

Ключевые слова: жидкостный ракетный двигатель, теплообмен, численные методы.

Введение. На сегодняшний день численное моделирование широко применяется в инженерной практике для расчета сложных физических процессов. Это связано прежде всего с возможностью замещения реальных экспериментов виртуальными.

Одной из наиболее трудоемких задач в проектировании ЖРД, требующей серьезной отработки, является обеспечение надежного охлаждения камеры двигателя [1]. Уменьшить материальные и временные затраты, возникающие при решении данной проблемы, можно с помощью совместного численного моделирования течения газа в камере двигателя и ее охлаждения.

Постановка задачи. Целью данной работы является построение вычислительной модели стационарного процесса в охлаждаемой камере ЖРД для определения параметров газового потока продуктов сгорания, тепловых потоков, направленных от горячих газов в стенку камеры, температуры стенки камеры и температуры жидкости в охлаждающем тракте.

() Дубровский И.Д., Бучарский В.Л., 2018
Методика моделирования. Решение поставленной задачи было разделено на несколько последовательных этапов:

1. Выбор математической модели для описания процессов в камере сгорания ЖРД.

2. Выбор методов решения уравнений математической модели.

3. Моделирование процесса охлаждения и сравнительный анализ полученных результатов со стандартной методикой расчета охлаждения [2].

Математическая модель. Схематично протекание процесса теплообмена в камере ЖРД можно представить следующим образом: тепло путем конвекции и лучеиспускания передается от горячих продуктов сгорания стенке камеры сгорания. С помощью теплопроводности тепло передается через стенку и далее путем конвекции - в охлаждающую жидкость. Наружная поверхность несущей стенки камеры полностью теплоизолирована [2].

Для описания данного процесса в качестве основной расчетной модели была выбрана модель идеального сжимаемого 
газа, описываемая нестационарными гиперболическими уравнениями неразрывности, сохранения количества движения, сохранения энергии и дополненная уравнением Менделеева - Клапейрона [3; 4]. Все вышеперечисленные газодинамические уравнения сохранения были получены для одномерного потока продуктов сгорания в камере ЖРД. Для удобства проведения численных расчетов вышеперечисленная система уравнений была записана в следующей векторной форме (ось ОХ проходит вдоль оси камеры сгорания, начало координат размещается в точке положения фронта пламени):

$$
\begin{gathered}
\frac{\partial \boldsymbol{U} \cdot F}{\partial t}+\frac{\partial \boldsymbol{E} \cdot F}{\partial x}=\boldsymbol{B} \\
\boldsymbol{U}=\left(\begin{array}{l}
\rho \\
\rho w \\
\rho e
\end{array}\right), \boldsymbol{E}=\left(\begin{array}{l}
\rho w \\
\rho w^{2}+p \\
(\rho e+p) w
\end{array}\right), \boldsymbol{B}=\left(\begin{array}{l}
0 \\
p \frac{\partial F}{\partial x} \\
-q P
\end{array}\right) .
\end{gathered}
$$

Здесь:

$\boldsymbol{\rho}$ - плотность, $\boldsymbol{p}$ - давление, $\boldsymbol{w}$ скорость, $\boldsymbol{e}-$ удельная внутренняя энергия, $\boldsymbol{q}$ - тепловой поток от наружной стенки камеры в жидкость, $\boldsymbol{F}$ - площадь поперечного сечения камеры сгорания, $\boldsymbol{P}$ периметр камеры.

Движение

охладителя в

зарубашечном тракте в соответствии со стандартной методикой расчета [2] описывалось одномерным стационарным уравнением сохранения внтуренней энергии с учетом притока тепла, полученным методом контрольного объема, и одномерным стационарным уравнением неразрывности $[3 ; 4]$ :

$$
\begin{aligned}
& \frac{\partial(\dot{m} u)}{\partial x}+q P=0, \\
& \rho w F=\dot{m}=\text { const. }
\end{aligned}
$$

Здесь:

$\boldsymbol{u}-$ внутренняя энергия жидкости, $\boldsymbol{\dot { m }}-$ массовый расход охладителя, $\boldsymbol{q}$ - тепловой поток от наружной стенки камеры в жидкость, $\boldsymbol{P}$ - периметр камеры, $\boldsymbol{\rho}$ плотность охладителя, $\boldsymbol{w}$ - скорость охладителя, $\boldsymbol{F}$ - площадь поперечного сечения тракта охлаждения.

Теплообмен между продуктами сгорания в камере и «горячей» стороной стенки, а также «холодной» стороной стенки и охлаждающей жидкостью, описывался на основании закона Ньютона Рихмана, где коэффициент теплоотдачи от «холодной» стороны стенки к охлаждающей жидкости определялся на базе эмпирических зависимостей Михеева с учетом оребрения охлаждающего тракта [2; 5], a коэффициент теплоотдачи от продуктов сгорания к «горячей» стороне стенки вычислялся с помощью зависимости Гухмана - Илюхина [2]:

$$
\alpha_{ж . p .}=87.9 \eta_{p} Z \beta\left(\frac{\dot{m}_{\text {охл }}}{F_{\text {охл }}}\right)^{0.8}\left(\frac{1}{d_{\text {экв }}}\right)^{0.2},
$$

$$
\alpha_{2}=\frac{0.026 \dot{m}^{0.82} C_{p}\left[T_{2 . c m}\right] \mu\left[T_{2 . c m}\right]^{0.18}}{d^{1.82}}\left(\frac{T_{\kappa}}{T_{2 . c m}}\right)^{0.35} \text {. }
$$

Здесь:

$\boldsymbol{\eta}_{\boldsymbol{p}}$ - коэффициент оребрения тракта, $\boldsymbol{Z} \boldsymbol{\beta}$ - коэффициент, учитывающий физические свойства охладителя, $\boldsymbol{d}_{\text {эк }}-$ эквивалентный диаметр охлаждающего тракта, $\dot{\boldsymbol{m}}_{\boldsymbol{\prime} \boldsymbol{x}}-$ массовый расход охладителя, $\boldsymbol{F}_{\text {охл }}$ - площадь поперечного сечения охлаждающего тракта, $\boldsymbol{q}$ - тепловой поток от продуктов сгорания в стенку камеры, $\dot{\boldsymbol{m}}$ - массовый расход продуктов сгорания, $\boldsymbol{T}_{\text {z.cm }}$ - температура «горячей» стороны стенки, $\boldsymbol{C}_{\boldsymbol{p}}\left[\boldsymbol{T}_{\mathbf{z . c m}}\right]$ - удельная теплоемкость при постоянном давлении продуктов сгорания, вычисленная при температуре стенки, $\boldsymbol{\mu}\left[\boldsymbol{T}_{\text {z.cm }}\right]$ - динамическая вязкость продуктов сгорания, вычисленная при температуре стенки, $\boldsymbol{T}_{\boldsymbol{\kappa}}-$ температура торможения в камере сгорания, $\boldsymbol{d}$ - диаметр камеры сгорания.

Удельная теплоемкость при постоянном давлении и динамическая вязкость продуктов сгорания были вычислены для постоянного, «замороженного» состава продуктов сгорания, соответствующего камере сгорания.

Процесс теплопроводности через стенку рассчитывался с помощью закона Фурье [6]; была учтена зависимость коэффициента теплопроводности от средней температуры стенки. Температура охлаждающей жидкости определялась из решения уравнения энергии. Наружная 
стенка камеры предполагалась теплоизолированной от окружающей среды; учитывалась зависимость удельной теплоемкости жидкости от средней температуры в ядре потока [7].

\section{Метод решения уравнений} математической модели. Для интегрирования системы уравнений (1) был использован метод конечных объемов [8]. Для этого расчетная область была разбита на $N$ конечных объемов и система уравнений (1) была записана в интегральной форме для каждого из них

$$
\frac{\partial \widetilde{\boldsymbol{U}}_{i} \cdot \widetilde{F}_{i}}{\partial t}=-\frac{1}{h_{i}}\left(\boldsymbol{E}_{i+1 / 2} \cdot F_{i+1 / 2}-\boldsymbol{E}_{i-1 / 2} \cdot F_{i-1 / 2}\right)+\widetilde{\boldsymbol{B}}_{i}
$$

Как известно, одним из основных этапов метода контрольного объема есть реконструкция параметров потока по их средним по контрольному объему значениям [9]. В настоящей работе использовалась оригинальная явная полиномиальная компактная реконструкция третьего порядка точности по пространству. В предлагаемом подходе, в отличие от рассмотренного в работе [10], в качестве дополнительных переменных используются не средние в конечном объеме значения производных реконструируемой функции (далее функции), а значения некоторых дифференциальных операторов этой функции на границах контрольного объема. В качестве таких операторов были выбраны разложения в ряд Тейлора разделенных разностей средних значений функции соответствующего порядка точности.

Ниже приведены расчетные формулы для определения коэффициентов полинома второй степени, что соответствует третьему порядку точности аппроксимации (начало локальной системы координат расположено в центре конечного объема):

$$
\left.\begin{array}{l}
\int_{h_{i} / 2}^{h_{i}} P_{2}(x) d x=\tilde{u}_{i} h_{i} \\
\left.\frac{d P_{2}(x)}{d x}\right|_{x=\frac{h}{2}}=\Lambda_{i+1 / 2}^{1}\left(\tilde{u}_{i}\right) \\
\left.\frac{d P_{2}(x)}{d x}\right|_{x=-\frac{h}{2}}=\Lambda_{i-1 / 2}^{1}\left(\tilde{u}_{i}\right)
\end{array}\right\},
$$

Здесь:
$P_{2}(x)=a 0+a_{1} x+a_{2} x^{2}-$ искомый полином,

$\tilde{u}_{i}=\int_{-h_{i} / 2}^{h_{i} / 2} u(x) d x \quad-\quad$ среднее значение реконструируемой функции,

$\Lambda_{i+1 / 2}^{1}(\tilde{u})=\frac{\tilde{u}_{i+1}-\tilde{u}_{i}}{0.5\left(h_{i}+h_{i+1}\right)} \quad-\quad$ разделённая разность.

После определения коэффициентов полинома значения функции на границах конечного объема определяются по следующим зависимостям:

$$
\begin{aligned}
& u_{i+1 / 2}^{-}=\tilde{u}_{i}+\frac{h}{6}\left(\Lambda_{i-1 / 2}^{1}(\widetilde{u})+2 \Lambda_{i+1 / 2}^{1}(\tilde{u})\right), \\
& u_{i-1 / 2}^{+}=\tilde{u}_{i}-\frac{h}{6}\left(2 \Lambda_{i-1 / 2}^{1}(\widetilde{u})+\Lambda_{i+1 / 2}^{1}(\widetilde{u})\right) .
\end{aligned}
$$

Как видно, расчетные формулы являются компактными и явными, что упрощает алгоритм и существенно сокращает время проведения расчетов.

Приведенная процедура реконструкции была использована в следующей методике интегрирования системы уравнений (6) методом конечного объема [10]:

1) вычисляются разделенные разности;

2) по зависимостям (8) определяются реконструированные значения искомых функций на границах конечных объемов;

3) численные потоки на границах конечных объемов определяются с помощью процедуры Лакса - Фридрихса приближенного решения задачи Римана [10];

4) после определения численных потоков система уравнений интегрируется по времени с помощью трехшаговой SSP схемы Рунге - Кутта [11].

$$
\begin{aligned}
& T_{\text {жi }}=T_{\text {жi }+1}+\Delta h \frac{q P}{\dot{m} C\left[T_{\text {ж.cp }}\right]}, \\
& T_{\varkappa . c m}^{n+1}=T_{\varkappa}^{n}+\frac{q F_{\text {бок }}}{\alpha_{\varkappa . p .}}, \\
& T_{2 . c m .}^{n+1}=T_{\text {ж.. } c m}^{n}+\frac{q \delta}{\lambda\left[T_{\text {cm.cp. }}^{n}\right]} .
\end{aligned}
$$


Для вычисления температуры охладителя осуществлялась дискретизация уравнения сохранения внутренней энергии по явной схеме Эйлера [12]. Таким образом, были получены следующие расчетные выражения для определения температур жидкости, «холодной» и «горячей» сторон стенки камеры.

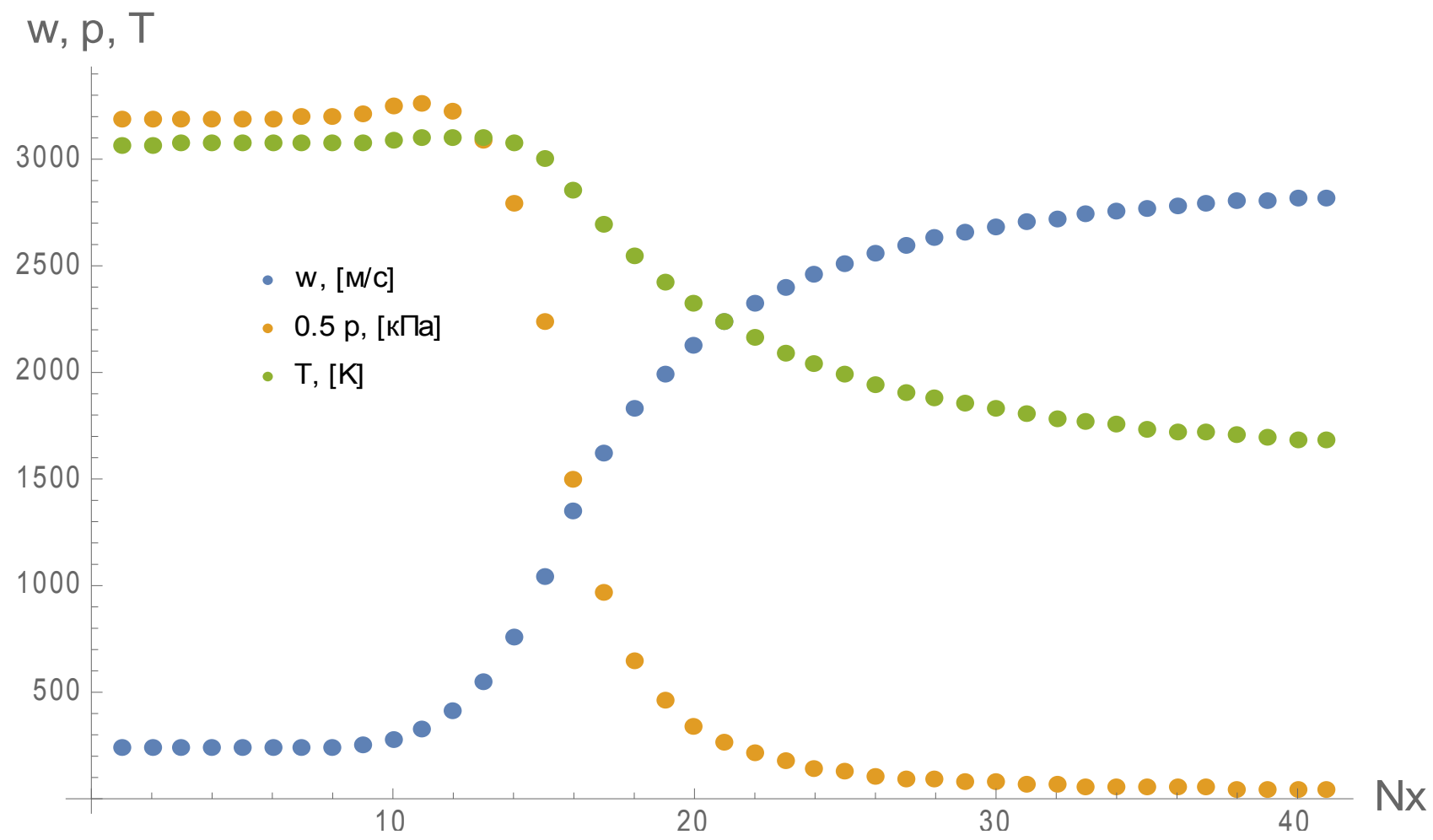

Рис 1. Распределение газодинамических параметров по длине камеры

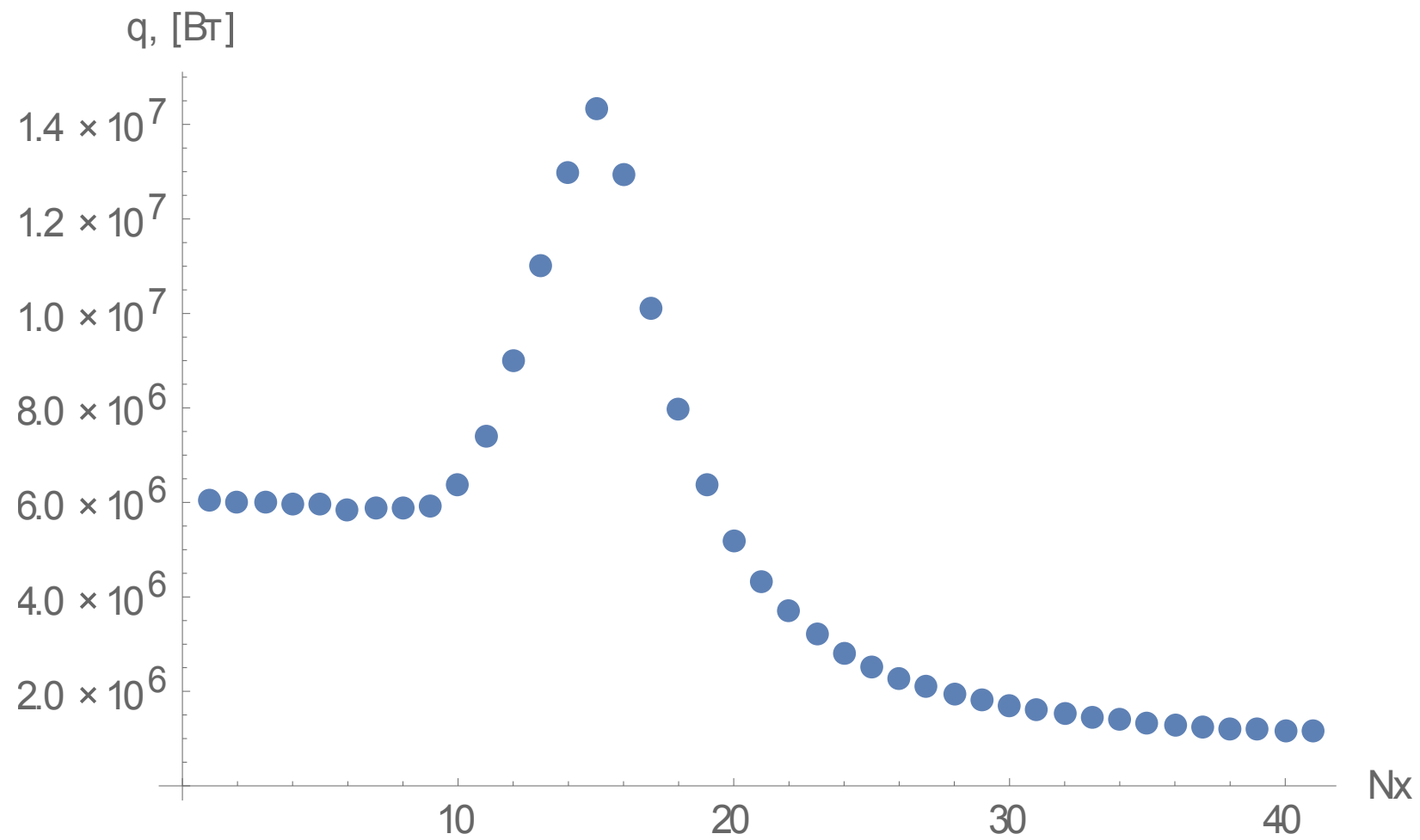

Рис. 2. Распределение удельных тепловых потоков по длине камеры 


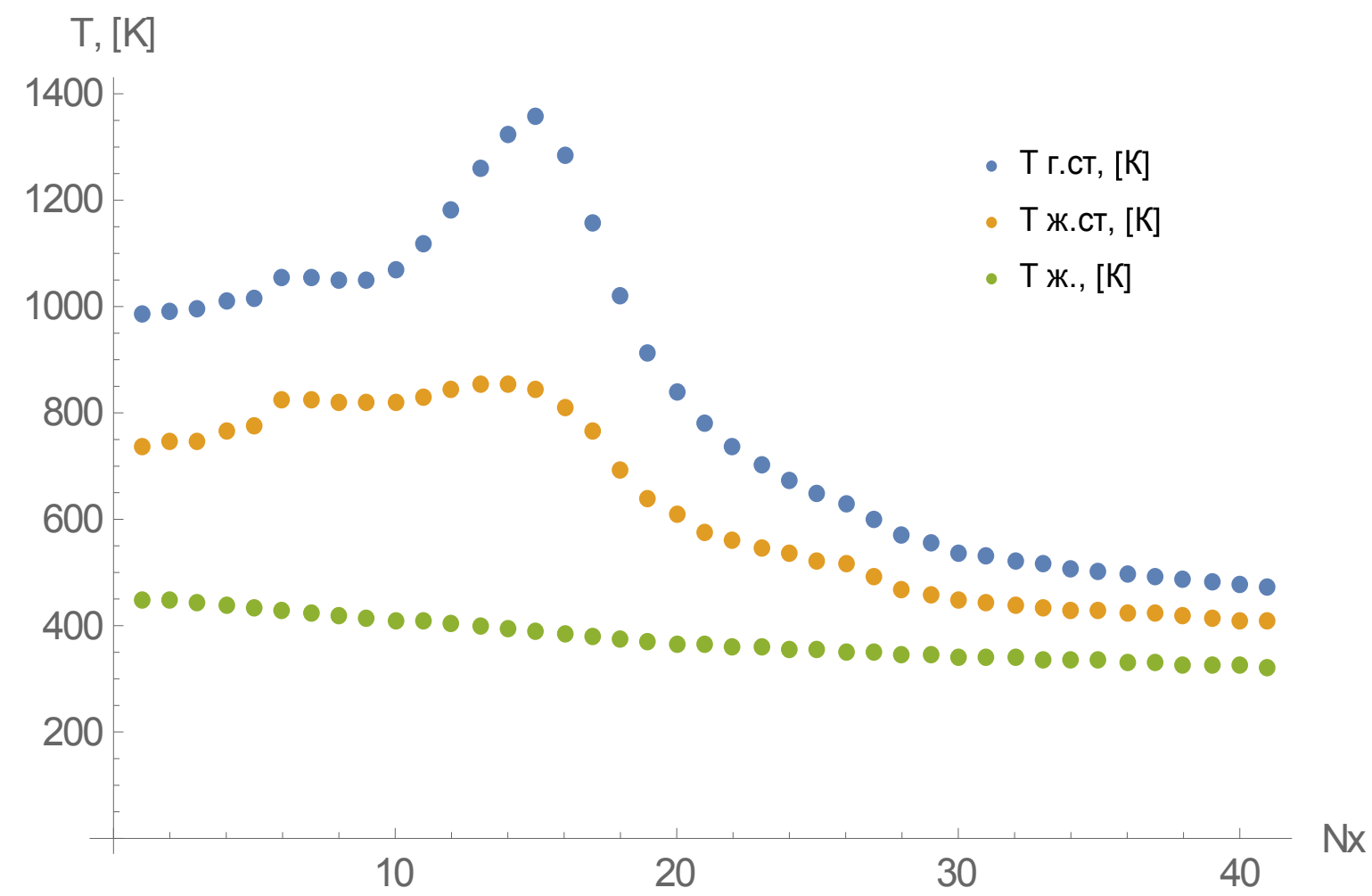

Рис 3. Распределение температур по длине камеры

Здесь:

$\boldsymbol{T}_{\text {ж. }}$ - температура жидкости в і-м сечении

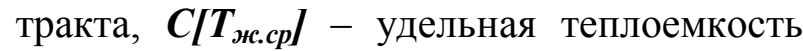
жидкости, как функция средней температуры жидкости, $\lambda\left[\boldsymbol{T}_{\text {cm.cp }}\right]$ коэффициент теплопроводности стенки камеры, как функция средней температуры стенки, $\dot{\boldsymbol{m}}$ - массовый расход охладителя, $\boldsymbol{F}_{\text {бок }}$ - боковая площадь камеры, $\boldsymbol{q}$ тепловой поток от продуктов сгорания в стенку камеры, $\boldsymbol{\delta}$ - толщина стенки камеры, $\boldsymbol{n}+\boldsymbol{1}$ - новый шаг по времени, $\boldsymbol{T}_{\text {z.cm }}$ - температура стенки со стороны газа, $\boldsymbol{T}_{\text {ж.cm }}$ - температура стенки со стороны жидкости, $\boldsymbol{\alpha}_{\text {ж.p. }}-$ коэффициент теплоотдачи от стенки к жидкости, $\boldsymbol{P}$ - периметр камеры.

Результаты и обсуждение. Для осуществления численных расчетов камера была разбита по длине на $\mathrm{N}=40$ конечных объемов равномерным шагом $\boldsymbol{\Delta} \boldsymbol{h}$. Шаг по времени $\boldsymbol{\Delta} \boldsymbol{\tau}^{\boldsymbol{n}}$ выбирался в соответствии с условием устойчивости Куранта для $\boldsymbol{n}$-й итерации цикла:

$$
\Delta \tau^{n} \leq \varepsilon \frac{\Delta h}{\operatorname{Max}\left[\left|w_{i}^{n}\right|+a_{i}^{n}\right]} .
$$

Здесь:

$\boldsymbol{w}_{\boldsymbol{i}}$ - скорость потока в $\boldsymbol{i}$-м сечении камеры, $\boldsymbol{a}_{\boldsymbol{i}}-$ местная скорость звука в $\boldsymbol{i}$-м сечении камеры, $\boldsymbol{\varepsilon}<\boldsymbol{1}-$ коэффициент запаса устойчивости разностной схемы.

В результате были получены кривые распределения газодинамических параметров, температур охладителя, «горячей» и «холодной» сторон стенки камеры. Для проверки полученных результатов был выполнен расчет охлаждения по стандартной методике [2] с дальнейшим сопоставлением полученных результатов по обеим методикам. Были получены следующие отклонения:

Расхождения основных параметров, определяемых в расчете охлаждения

\begin{tabular}{|l|l|l|}
\hline Параметр & $\begin{array}{l}\text { Среднеинтегральное } \\
\text { абсолютное отклонение }\end{array}$ & $\begin{array}{l}\text { Относительное } \\
\text { среднеинтегральное } \\
\text { отклонение, \% }\end{array}$ \\
\hline $\mathrm{q},[\mathrm{MBT}]$ & 1.05 & 7.07 \\
\hline $\mathrm{T}_{\text {ж.ст., }[\mathrm{K}]}[\mathrm{K}]$ & 88.13 & 6.43 \\
\hline $\mathrm{T}_{\text {г.с.. }}[\mathrm{K}]$ & 6.06 & 6.47 \\
\hline $\mathrm{T}_{\text {ж, }}[\mathrm{K}]$ & 5.46 & 1.23 \\
\hline
\end{tabular}


Максимальное расхождение между параметрами наблюдалось в критическом сечении камеры в большую сторону относительно стандартной методики.

Выводы. В данной работе приведено численное решение системы одномерных дифференциальных уравнений газовой динамики и теплового баланса для камеры ЖРД. В результате расчета в каждом сечении камеры определены параметры газового потока продуктов сгорания, удельные тепловые потоки, температура стенки камеры и температура жидкого охладителя.

Расхождение со стандартной методикой обусловлено учетом распределения газодинамических параметров по длине камеры сгорания и их влияния на процесс охлаждения. Таким образом, можно сделать вывод, что в данной работе получены более точные результаты по сравнению с расчетом охлаждения, выполненного с помощью стандартной методики.

\section{Библиографические ссылки}

1. Теория ракетных двигателей : учебник для студ. машиностроительных спец. вузов. Под ред. В. П. Глушко, В. Е. Алемасова, А. Ф. Дрегалина, А. П. Тишина. Москва : Машиностроение, $1980.533 \mathrm{c}$.

2. Добровольский М. В. Жидкостные ракетные двигатели. Москва : Машиностроение, 1968. 396 с.

3. Лойцянский Л. Г. Механика жидкости и газа. Москва : Дрофа, 2003. $840 \mathrm{c}$.
4. Кочин Н. Е., Кибель И. А., Розе Н. В. Теоретическая гидромеханика. В 2-х ч. Ч. 2. Москва : Физматгиз, 1963. $728 \mathrm{c}$.

5. Васильев А. П. Основы теории и расчета жидкостных ракетных двигателей. Под ред. В. М. Кудрявцева. Москва : Высш. школа, 1983. 703 с.

6. Михеев М. А. Основы теплопередачи. Москва : Энергия, 1977. $345 \mathrm{c}$.

7. Варгафтик Н. Б. Справочник по теплофизическим свойствам газов и жидкостей. Москва : Наука, 1972. 721 с.

8. Андерсон Д., Таннехил Дж., Плетчер Р. Вычислительная гидромеханика и теплообмен. Пер. с англ. В 2-х т. Т. 1. Москва : Мир, 1990. 384 с.

9. Changqing $\mathrm{Hu}$, Chi-Wang Shu. Weighted Essentially Non-oscillatory Schemes on Triangular Meshes. Journal of Computational Physics. Vol. 150. Issue 1. 1999. P. 97-127.

10. Wang, Qian \& Ren, Yu-Xin \& Lee, Marka (2016). Compact high order finite volume method on unstructured grids I: Basic formulations and one-dimensional schemes. Journal of Computational Physics. 314. 10.1016/j.jcp.2016.01.036.

11. Gottlieb S. and Shu C.-W. Total variation diminishing Runge-Kuttaschemes: Math. Comp., 67. 1998. P. 73-85.

12. Калиткин Н. Н. Численные методы. Москва : Наука, 1978. 512 с.

Надійшла до редколегії 29.05.2018 р.

УДК 629.7.036.54

\title{
ПЕРСПЕКТИВНІ НАПРЯМКИ НАУКОВИХ ДОСЛІДЖЕНЬ НА КАФЕДРІ ДВИГУНОБУДУВАННЯ
}

\author{
М. О. Катренко, С. Г. Бондаренко, О. В. Сосновська,
} О. С. Золотько, О. Л. Марченко, С. М. Подольчак

Дніпровський наџіональний університет імені Олеся Гончара, пр. Гагаріна, 72, м. Дніпро, 49010, Україна, e-mail: arush@ua.fm

Подано ретроспективу напрямків наукових досліджень співробітників кафедри двигунобудування фізико-технічного факультету. Розглянуто історичне становлення та розвиток, здобутки вчених кафедри в галузі ракетного двигунобудування, а також перспективи їх подальших досліджень.

Ключові слова: напрямок, перспективи, дослідження, ракетні двигуни, актуальність. 